\title{
Cervical Cancer in Women with Inflammatory Pap Smears
}

\author{
Mongia Achour ${ }^{1,2^{*}}$, Dorra Zeghal ${ }^{3}$ \\ ${ }^{1}$ IMEC (Immuno-Microbiology Environmental and Carcinogenesis) UNIT, Faculty of Sciences, Bizerta, Tunisia; ${ }^{2}$ Higher Institute of \\ Biotechnology of Béja, Béja, Tunisia; ${ }^{3}$ Center of Maternity and Neonatology, Service of Gynecology and Obstetrics, Tunis, Tunisia. \\ Email: ${ }^{\text {mahaachour2000@yahoo.fr }}$
}

Received August $11^{\text {th }}, 2013$; revised September $11^{\text {th }}$, 2013; accepted September $20^{\text {th }}, 2013$

Copyright (C) 2014 Mongia Achour, Dorra Zeghal. This is an open access article distributed under the Creative Commons Attribution License, which permits unrestricted use, distribution, and reproduction in any medium, provided the original work is properly cited. In accordance of the Creative Commons Attribution License all Copyrights (C) 2014 are reserved for SCIRP and the owner of the intellectual property Mongia Achour, Dorra Zeghal. All Copyright (c) 2014 are guarded by law and by SCIRP as a guardian.

\section{ABSTRACT}

In spite of preventive measures such as Papanicolaou cervical cytological analysis and, more recently, vaccination against HPV infection, cancer of the uterine cervix continues to be one of the most frequent causes of mortality among women worldwide, particularly in developing countries. In this prospective study, sixty patients with inflammatory Pap smears had a colposcopy with directed biopsies. The average age of our patients was 42 years. Results showed that colposcopy is normal in $10 \%$ of women. It showed normal transformations, ectropion, a colpotis and polyp at $8.33 \%, 21.66 \%, 13.33 \%$ and $5 \%$ respectively. It was able to detect changes with Grade I atypical transformations (28.33\%), and Grade II atypical transformations in $13.33 \%$ of cases. The biopsies were objectified dysplasia and carcinoma in $\mathbf{2 4 . 1 3 \%}$ of cases with carcinoma in situ, micro invasive squamous cell carcinoma and invasive carcinoma glandular. Moreover, we detected HPV-specific antibodies in sera of these patients. Results showed that six patients $(10 \%)$ showed a positive reactivity to at least one of the HPV-16 or HPV-18 antigens and sera showed different reactivity to the different antigens with the following percentages: 5\%, 3\%, 2\%, 3\% and 3\% for L1 HPV-16, E6 HPV-16, E7 HPV-16, E6 HPV-18 and E7 HPV-18 respectively. Among patients having positive antibody response, $83.33 \%$ were cases of dysplasia and carcinoma. We concluded that the Pap smear, examination of key screening for cervical cancer, is a screening test without diagnostic value and more specifically any inflammatory Pap smear should be considered a positive test and led to further investigations. Moreover, colposcopy is an exam that is performed on an outpatient basis; it allows a detailed study of the cervix and reduces the negative rate of cytology. In addition, early detection of HPV antibodies could help the follow-up of patients.

\section{KEYWORDS}

Pap Smear; Inflammatory; Cervical Cancer; Human Papillomavirus

\section{Introduction}

Cervical cancer has been the subject of several epidemiological studies since 150 years ago. It is among the first two gynecological cancers in countries development and developed countries of the flowering of sexually transmitted diseases and represents a public health problem. It is a leading cause of death among women between 35 and 45 years [1]. Cervical cancer is the second most common cancer in females in the world with around 500,000 new cases occurring annually, but the first in the developing countries with a high mortality if not diag-

"Corresponding author. nosed early [2].

In Tunisia, cervical cancer is the second most frequent cancer among women. Previous study was undertaken to determine epidemiological and pathological profile of cervical cancer in Tunisia, which shows that the incidence of cervical cancer in our country is relatively low in spite of the absence of a screening program. Moreover, authors have reported that squamous carcinoma was more frequent than adenocarcinoma [3]. In fact, in our country, the incidence of cervical cancer is inferior to 5/100,000 women, and the viral positivity type HPV-16 is the most common for all studies in Tunisia [4]. The incidence of cervical cancer is estimated to 11.2 per 
100,000 women in developed countries, and 18.2 per 100,000 women in developing countries and the incidence was different when studying developing areas, country by country [5].

In spite of preventive measures such as Papanicolaou cervical cytological analysis and, more recently, vaccination against HPV infection, cancer of the uterine cervix continues to be one of the most frequent causes of mortality among women worldwide, particularly in developing countries [6]. The etiological role of human papillomavirus (HPV) infection in cervical precancer/cancer is supported by numerous biological and epidemiological studies [7,8]. HPV 16 is the most common oncogenic type and is detectable in approximately half of all cervical $[9,10]$. HPV 18 is the second most common type in invasive cervical cancer world-wide [10].

We aimed in this study to show that the Pap smear, examination of key screening for cervical cancer, is a screening test without diagnostic value and more specifically any inflammatory Pap smear should be considered a test positive and led to further investigations, as well as to emphasize the importance of colposcopy to vaginal inflammatory cervical smears. Moreover, we used a GSTcapture ELISA to detect antibodies to different HPV 16 and HPV 18 antigens.

\section{Materials and Methods}

\subsection{Cytology-Colposcopy-Histology Tests}

We conducted a prospective study on the cyto-colpohistological comparison of inflammatory Pap smear screening tests performed in patients who consulted "C" service center of maternity and neonatology of Tunis. The study included 60 inflammatory smears; all patients had a colposcopy and 58 of them have benefited from examined biopsies. The study involved women with genital activity, perimenopausal and postmenopausal women. We evaluated the sensitivity of Pap smear in screening for cervical cancer. We have stressed the value of colposcopy in addition to cytology even in the absence of signs for a dysplasia. We correlated the results of various tests:

- Cytology.

- Colposcopy (indirect signs suggestive of cervical lesions).

- Histology (biopsies).

All Pap smears were performed on an outpatient basis. The cytologic interpretation is provided by pathology services of the hospital "La Rabta" and "Salah Azaiz" Institute. Results are then collected and placed in the records of the outpatient.

Women with inflammatory Pap smears were called for colposcopy within the service itself. Colposcopy was performed in room colposcopy without anesthesia, by four different Colposcopists medical team. In the absence of evidence in favor of a lower genital tract infection, colposcopy is done immediately.

Results of colposcopy are expressed according to the classification of the French society of colposcopy. Directed biopsies were performed in the presence of anomalies detected by colposcopy. Histological examination is provided by the pathology laboratory of the hospital "La Rabta".

\subsection{Recombinant Proteins and GST-Capture ELISA}

HPV-types 16 and 18 E6 and E7 as well as HPV-16 L1 have been over expressed as fusion proteins with glutathione S transferase (GST) in E. coli BL21 as previously described [11].

HPV-types 16 and 18 E6 and E7 coding sequences fused at their 3'-end in frame to a sequence encoding the terminal undecapeptide of the SV40 (tag), were isolated from bluescript plasmids described by Meschede et al. [12] and inserted into a pGEX vector for expression as GST fusion proteins in E. coli.

E. coli BL21 cells transformed with the pGEX plasmids were grown in Luria Bertani medium containing 1 $\mathrm{mm}$ ampicillin. Recombinant protein expression was induced by adding $0.25 \mathrm{~mm}$ isopropyl- $\beta$-D-thio-galactoside (IPTG) to the medium. Bacteria were harvested and then pelleted bacteria were resuspended with complete protease inhibitor and lysed with a high-pressure homogenizer. Lysates were cleared and stored at $-20^{\circ} \mathrm{C}$. The lysate's total protein concentration was determined using the Bradford-Reagent.

\section{Human sera.}

Blood samples were collected from patients. As controls, we have chosen seventy healthy women, randomly taken out of the general adult women population of Tunisia. The status of patients was clinically determined based essentially on cytology and biopsy analysis. Sera were collected and conserved at $-20^{\circ} \mathrm{C}$ until use.

\section{GST-capture ELISA test.}

Polysorp plates were coated overnight, with glutathione casein to $2 \mathrm{mg} / \mathrm{ml}$ in coating buffer, then were blocked with $180 \mathrm{ml}$ of casein blocking buffer per well and incubated $1 \mathrm{~h}$ at $37^{\circ} \mathrm{C}$. After washing, wells were incubated for $1 \mathrm{~h}$ at room temperature, with the cleared lysates from $E$. coli over expressing GST fusion proteins, diluted in casein blocking buffer $(0.2 \%(\mathrm{w} / \mathrm{v}))$ casein in phosphate buffer saline to $0.25 \mathrm{mg} / \mathrm{ml}$ total lysate protein.

After washing, the ELISA plates were incubated with serum diluted 1/200 in buffer. Bound human antibodies were detected by immunoglobulin Ig G goat anti-Human biotinylated, at a dilution of $1 / 10^{5}$ and the streptavidine conjugated to horseradish peroxydase (HRP) diluted at 
$1 / 10^{4}$ and incubated for $1 \mathrm{~h}$ at room temperature. As substrate we use the tetramethylbenzidine. The reaction was stopped after $7 \mathrm{~min}$, by adding $50 \mathrm{ml}$ of $1 \mathrm{M}$ sulphuric acid/well. Thereafter, the absorbance at $450 \mathrm{~nm}$ was measured; the absorbance in wells with GST alone as antigen defined the background reactivity of a serum and can be subtracted from the absorbance with the GST fusion protein to calculate the specific reactivity of a serum against the fused antigen. The cut-off value was calculated as previously described [11].

\section{Results}

\section{Results of Cytological Examination}

Cytology examination of the Pap smears showed that the 60 samples are inflammatory. Inflammatory Pap is defined by the presence of magnification $(\times 400)$ of 10 inflammatory cells per field (Polynuclear NeutrophilsHistiocytes-Plasmocytes and Lymphocytes).

The criteria of inflammation are the abundance of inflammatory cells and the presence of altered polymorphonuclear. Sometimes identifies a specific pathogen:

- Fungal inflammatory smear.

- Inflammatory smears with trichomonas.

- Condyloma.

- Inflammatory smears with herpes simplex.

- Other smears consisting of metaplasia and non-specific inflammatory smears, cytolytic and hemorrhagic.

An inflammatory process related to a specific or not can be argued before the infection following changes:

- The presence of numerous trails Neutrophils often altered.

- False Eosinophilia.

- The presence of perinuclear halos.

- Swelling of the core becomes large with, however, a fine chromatin and nuclear membrane.

Inflammatory samples, always have a "dirty" appearance with a background not optically empty, but rich in inflammatory cellular elements with possibly or pathogens, and secretion and mucus.

As we can see in Figure 1, colposcopy applied for the sixty cases confirm the existence of different profiles (normal colposcopy, ectropion, colpites, polyp and atypical transformation).

- For normal colposcopy, the connective tissue is well vascularized, the liquid interstitial is abundant and the collagen fibers are dispersed.

- Normal transformation area: during the redesign process appear different colposcopic aspects corresponding to the progression of squamous and glandular elements of regression.

- In the case of ectropion, there was eversion of the cervical canal and the glandular mucosa was secondarily externalized. This eversion has a diameter that ex-



Figure 1. Results of colposcopy (percentage) for the sixty cases tested. (a) Normal colposcopy; (b) Isolated ectropion; (c) Colpites; (d) Normal transformation area; (e) Cervical polyp; (f) Atypical transformation grade I; (g) Atypical transformation grade II.

ceeds 1 centimeter long axis.

- Appearance colpitis translated certainly an acute inflammation of the lining, but it has no specific character. It is often more pronounced in parasitic trichomonas, but it can be also in fungal infections and pyogenic bacteria.

- A polyp has a connective axis and epithelial lining. The easiest polyp is a disc of larger than other glandular mucosa. The value of colposcopy is not to describe the coating of polyps, but to clarify whether this is polyps, look for associated injuries and set the terms of the treatment.

- Atypical transformation Grade I: the exam without preparation objective never congestive or erosive red areas, but sometimes leukoplakia. Areas mosaic or punctuation spontaneous end network. The blood vessels have a regular size and direction of the inter capillary distances capillaries are normal (less than 300 microns).

- Atypical transformation Grade II: They are usually due to dysplasia, but pregnancy and some infections can give the same aspects

We performed 58 biopsies on 60 colposcopic examinations. The indications were different. Biopsies ectropions and areas of normal processing were performed because of the extent of the injury or because there were signs of severe combined colposcopic.

Table 1 shows the histologic data for the biopsies tested. In fact, for confrontation, cytology-histology and inflammatory smears considered in our study, it has been revealed different figures and are distributed as follows. The majority of cases showed condyloma (19 cases), followed by cervicitis (16 cases) then moderated and severe dysplasia (11 cases).

Carcinoma cases are detected in only 2 cases (3.44\%).

As summarized in Table 2, for a total of 60 smear inflammatory lesions requiring the colposcopic directed biopsy (58 cases): 
- Histology is in favor of a benign lesion in $73.33 \%$ of cases.

- 3 cases of carcinoma were diagnosed with in situ carcinoma, micro-invasive squamous cell carcinoma and invasive glandular cancer.

Table 3 showed that the seroresponse against the different antigens L1, E6, E7 HPV-16 and E6, E7 HPV-18 determined using the GST-capture ELISA was more important for cases than for controls. The seropositivity toward the distinct antigens was different and it depends

Table 1. Histologic data of biopsies.

\begin{tabular}{ccc}
\hline & Number (n) Percentage (\%) \\
\hline Normal mucosa & 3 & 5.17 \\
Cervicitis & 16 & 27.58 \\
Condyloma & 19 & 32.75 \\
Condyloma acuminatum & 1 & 1.72 \\
Ectropion & 1 & 1.72 \\
Squamous metaplasia & 1 & 1.72 \\
Polyps & 1 & 1.72 \\
Mild dysplasia (INC1) & 3 & 5.17 \\
Moderate and severe dysplasia + CIN & 11 & 18.96 \\
(CIN2-CIN3-CIS) & & \\
Carcinoma & 2 & 3.44 \\
\hline
\end{tabular}

Table 2. Distribution of cases upon the analysis of histology of biopsies.

\begin{tabular}{ccc}
\hline & Cases (n) & Percentages \\
\hline Benign lesions & 44 & 73.33 \\
Low-grade lesions & 7 & 11.66 \\
High-grade lesions & 5 & 8.33 \\
$\quad(\mathbf{1}$ CIS) & 1 & \\
Squamous cell carcinoma & 1 & 1.66 \\
Glandular carcinoma & 1 & 1.66 \\
\hline
\end{tabular}

Table 3. Seropositivity against HPV-16 and HPV-18 antigens.

\begin{tabular}{ccc}
\hline Antigens & Case (n) & Percentage (\%) \\
\hline HPV16 L1 & 3 & 5 \\
HPV16 E6 & 2 & 3 \\
HPV16 E7 & 1 & 2 \\
HPV18 E6 & 2 & 3 \\
HPV18 E7 & 2 & 3 \\
\hline
\end{tabular}

on the type of antigen.

For the 60 inflammatory Pap smears considered in the present study, we detected HPV-specific antibodies in sera of these patients. Results showed that six patients (10\%) showed a positive reactivity to at least one of the HPV-16 or HPV-18 antigens and sera showed different reactivity to the different antigens. Higher prevalence is showed for L1 HPV16 antigen. Among patients having positive antibody response, (5/6: 83.33\%) were cases of dysplasia and carcinoma with 1 in situ carcinoma, 1 microinvasive squamous cell carcinoma and glandular invasive carcinoma.

Figure 2 gives different pictures for typical histology exalmination.

\section{Discussion}

Most developed countries have experienced in recent decades a significant decrease in invasive cervical cancers. This downward trend is mainly attributed to the beneficial effects of screening. This decrease can be quantified globally at $3 \%$ per year [13].

Our country is among the countries with a low incidence of cervical cancer, but it remains unfortunate prognosis in advanced stages, hence the importance of screening.

There is currently a sixty HPV which more than a dozen infect the genital epithelia. They are classified into two categories according to their integration in the cellular genome properties and their presence in invasive carcinomas [14]. Low risk HPV types $(6,11,42,44)$ and high risk HPV (16, 18, 31, 33, 35, 39, 51, 52, 56).

Richard monitoring of patients with dysplasia, calculated duration of progression to carcinoma in situ:

- 7 years on average to mild dysplasia.

- 3 years for moderate dysplasia.

- 1 year for severe dysplasia.

But in recent years several authors have found delays of any changes, so it is possible that HPV infection accelerates the progression.

Epidemiological study showed that the age of the patients regularly increases with the degree of atypia, which indicates a change in time [15].

The use of screening by cervical smear has reduced mortality from cervical cancer due to the long period of carcinogenesis and accessibility of cytological and histological detection of cervical cancer [16]. The interpretation of the Pap smear has changed a lot and reading criteria blades have been many changes, but the doctor continues to be solely responsible for the management and monitoring of abnormal smears.

Knowing that the false negative rate is on average 20\%, we believe that further investigations are necessary to remedy the deficiencies of cytology [17].

The false negative rate in our series is $39.28 \%$. False 


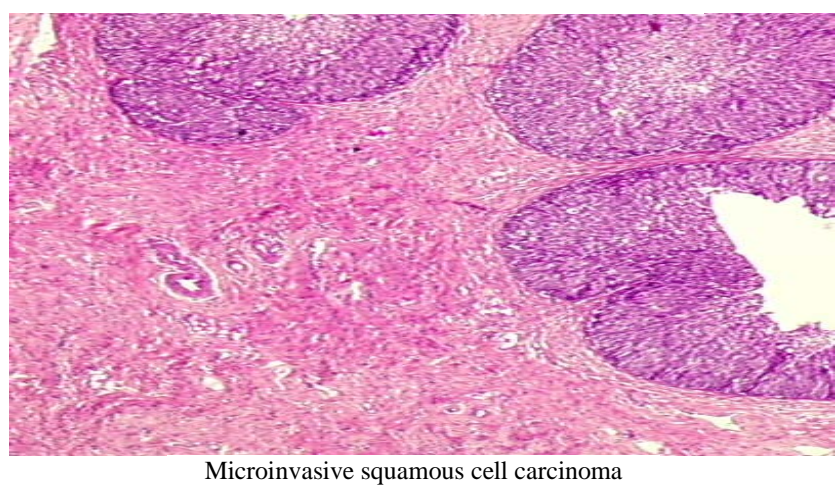

(a)



Endocervical adenocarcinoma (moderately differentiated infiltrating)

(c)

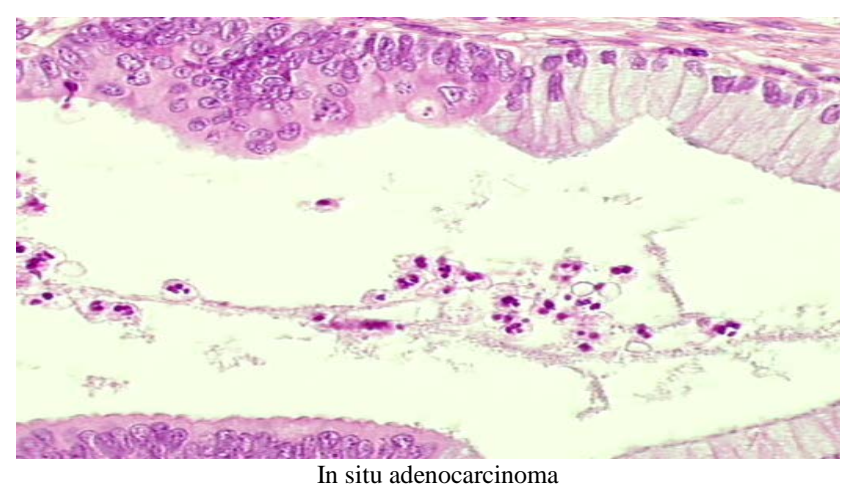

(b)

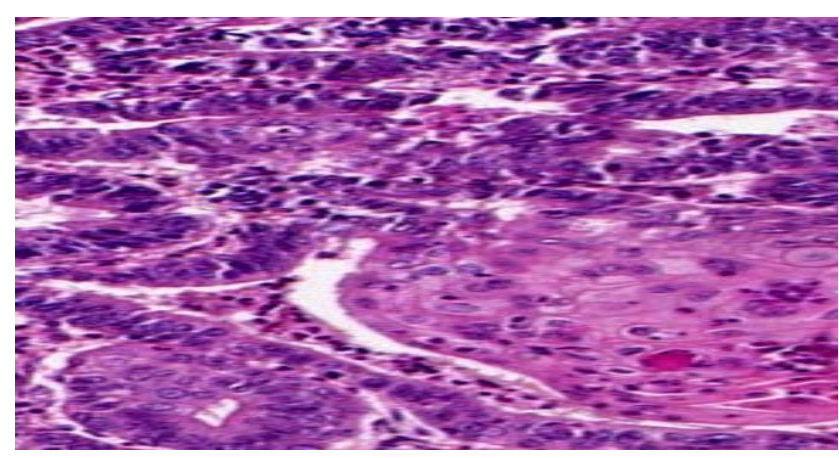

Glandular invasive carcinoma

(d)



(e)

Figure 2. Typical histology examination pictures. (a) Microinvasive squamous cell carcinoma; (b) In situ adenocarcinoma; (c) Endocervical adenocarcinoma (moderately differentiated infiltrating); (d) Glandular invasive carcinoma; (e) Endocervical dysplasia.

negatives are formidable and can have very serious consequences.

Pairmuti [18] in a study of 4781 women with precancerous or cancerous lesions of the cervix, counts $1.5 \%$ false negatives are as follows:

- In $58.5 \%$ of cases there was a sampling error.

- In $38.6 \%$ of cases of error screening.

- In $2.9 \%$ of cases of misinterpretation.

Vander Graaf [19] has taken over all smear slides initially classified I or II collected from patients who pre- sented within 3 years negative cytology moderate or severe dysplasia, carcinoma in situ or invasive carcinoma. The results are as follows:

- There was an underestimation of the damage in the first reading in $17.5 \%$ of cases.

- There is a good correlation in $70.2 \%$ of cases.

- $12.3 \%$ of smears were no significant.

Soot and Lauge [20] studied 748,871 smears in a population of 277,842 women in a period of ten years. They assess the reliability of cytology with strict criteria na- 
mely:

- Negative cytology is considered true negative if within 3 years it is followed by 2 negative cytology tests.

A positive cytology is validated if the histological evidence obtained in the year following the smear. So far, followed by successive smears has been the accepted method for monitoring Pap vaginal inflammation, but it may still miss some cytological abnormalities knowing dysplasia were found in the biopsies after review colposcopic on inflammatory smears [21,22].

Table 4 shows comparative findings for different authors. A study conducted by Jones and colleagues showed that the control of cervical smears by other inflammatory smear lower sensitivity and negative predictive value as colposcopy and directed biopsy. As against the specificity and positive predictive value are better. This means that vaginal Pap repetitive can still miss a lesion underlying the inflammation;

For Dargent [23], 77.6\% of false negative cytology recovered by colposcopy: is divided as follows:

- $14.2 \%$ for the CIN III (percentage of redressed diagnostics).

- $53.3 \%$ for the CIN II.

- $86.6 \%$ for the CIN I.

- $100 \%$ for plan condyloma.

Association cytology colposcopy is the best method for optimal sensitivity in cervical lesions.

In English literature [24,25,26], the incidence of HPV lesions, dysplasia, or even invasive cervical cancer, discovered by biopsies by colposcopy in patients with persistent inflammatory smears, between $18 \%$ and $35 \%$ [21].

Our Tunisian population is different from Western populations, due to customs and religious factors that are different. But according to a study made by the team Turkey N. C. SECKIN et al. in 1997 [27], the incidence of precancerous diseases and cancer of the cervix in their population that is similar to ours, in patients with Pap smears persistent inflammation is $22.7 \%$.

This rate is scary because it shows the large number of serious diseases is under-diagnosed.

So we think that inflammatory smears should not be considered a normal variant especially if it persists despite anti-infective therapy and should be considered a positive test to be completed by further investigations [28];

Colposcopy and directed biopsy seem essential not to ignore severe lesions.

To emphasize the value of colposcopy in vaginal Pap inflammatory, knowing that inflammation can hide intraepithelial lesions; A comparative study was conducted by Linda et al. comparing intraepithelial lesions found on histological study in women with inflammatory Pap smears and those with normal smears in two different populations: one exposed to sexually transmitted diseases and the other normal.

In the absence of inflammation, the possibility of finding intraepithelial lesions all stages taken during biopsies directed by colposcopy is the same in the population exposed to risk factors and which is not.

However, the rate of dysplasia doubles in case of inflammation in the population at risk, this is due to infections including HPV main factor for cervical cancer.

Campion, Singer and Mitchell [29] drew attention to a number of published studies including those of Kohan et al. [30] and Reiter et al. [26] showing that colposcopy and directed biopsies in cases of inflammatory Pap smear found:

- $30 \%$ of intraepithelial neoplasia after a single inflammatory smear.

- $70 \%$ of intra-epithelial neoplasia in cases of persistent inflammation.

This shows that the more severe the inflammation is so persistent, it hides most of the neoplastic cells. Nijirjesy's [31] study, in 1972, studied a group of 434 women with inflammatory Pap smears: 367 improved after treatment and 67 had persistent inflammation.

They have had a colposcopy with directed biopsies that found up to $78 \%$ of non-detected cervical cytology pathologies. The rate of false negative Pap smear in this

Table 4. Comparative findings for different authors.

\begin{tabular}{ccc}
\hline & Number of samples tested & Conclusion \\
\hline Pairmuti & 4781 (women with precancerous or cancerous lesions of the cervix) & True Negative cytology (if within 3 years it \\
is followed by 2 negative cytology tests) & 748,871 & $1.5 \%$ for the CIN III \\
Dargent & $77.6 \%$ of false negative (recovered by colposcopy) & $53.3 \%$ for the CIN II \\
& $86.6 \%$ for the CIN I & $100 \%$ for plan condyloma \\
\hline
\end{tabular}


study is $30 \%$. Sandmire [32] showed that $36 \%$ of patients with dysplasia see more severe lesions initially had inflammatory smears. In addition, it was found that 25 cases of patients with persistent inflammatory smears had the histological cervical cancer. Spitzer et al. [33] have tried to demonstrate this by a study published in 1997:

97 patients with initial inflammatory cervical smears, $56 \%$ have either a lesion HPV dysplasia or cervical cancer after histological examination of biopsies by colposcopy. He noted that the rate of false negatives in control smears reached 50\%. Kohan and Noumoff [34], published in 1985, a retrospective study over a period of 10 years in a New York hospital of 463 inflammatory Pap smears. We can conclude that the inflammatory Pap smears are not only due to infections and chronic cervicitis, as speculated Papanicolaou [35].

Pap smear, HPV DNA testing and colposcopy are complementary to each other for detecting preinvasive cervical cancer. HPV DNA is often transient in exfoliated cells or tissue and thus cannot provide a reliable indicator of past exposure [36]. HPV DNA detection is also limited by sampling difficulties because many unmarried women are unwilling to undergo gynecologic examinations for the collection of exfoliated cells, particularly in many regions of Asia, including China. Although a substantial proportion ( $\sim 50 \%)$ of women exposed to specific HPV types do not seroconvert [37], HPV antibody responses may be a useful proxy marker of cumulative exposure to HPV [38].

Serology is important in epidemiological studies. In fact, during natural infections with HPV, the humoral response mainly seems directed against the epitopes of capsid carried by the proteins L1 and L2 and certain early viral proteins (E2, E4, E6 and E7). Several types of antibodies are found with a title and a frequency of detection varying according to the type of infecting HPV and the recognized epitope which complicates their biological significance [39]. Previous seroepidemiological studies were carried out, from which we tried to reach a certain consensus in the interpretation and the diagnostic and prognostic interest of the antibodies. However, the detection of these various antibodies cannot be used as reliable marker of diagnosis or forecast. Indeed, the serum reflection of a viral infection always does not have a stable and single significance in clinical term, and the infections with HPV return within this framework. Only the search for neutralizing antibodies would have an interest to sign the curative phase of an infection with HPV [40], whereas the antibodies directed against the oncoproteins E6 and E7 of HPV-16 would be strongly associated to cervical cancer [41].

The cost of evaluating and treating the precursor lesions of uterine cervical cancer, of which many will not develop into invasive cancer, is high. According to Fol- len et al. (2005), this may be the reason why, in developing countries, cervical cancer goes undetected at higher frequencies than in developed countries [42].

Colposcopy is a test that is done on an outpatient basis, it helps to make a careful study of the cervix and to reduce the false negative rate of cytology.

\section{Conclusions}

Serology is not in itself a diagnostic method but must be kept in consideration for monitoring patients with a cervical smear inflammatory and most of them are detected in anti-HPV antibodies. Similarly, the determination of these antibodies by GST-capture ELISA and Luminex can help monitor vaccination programs on a large scale.

The histological and cytological colposcopic results allowed the therapeutic management of the cases analyzed. We proposed therapeutic modalities and monitoring strategies for patients based on the results of various examinations.

\section{Acknowledgements}

We would like to thank Professor ZOUARI Faouzia and Professor MAHJOUB Sami (Service of Gynecology and Obstetrics "C" Center of Maternity and Neonatology of Tunis) as well as all patients who participated in this study. We are grateful to Professor OUESLATI Ridha (IMEC: Immuno-Microbiology Environmental and Carcinogenesis Unit Faculty of Sciences of Bizerta) and Doctor Pawlita Michael from the DKFZ Germany for providing products used in the ELISA analysis.

\section{REFERENCES}

[1] M. Maâlej, A. Ben Youssef, M. Ben Andallah, et al., “Epidémiologie du Cancer du col de l’Utérus en Tunisie," Maghreb Médical, Vol. 242, 1991, pp. 12-17.

[2] H. Demirhindi, E. Nazlican and M. Akbada, "Cervical Cancer Screening in Turkey: A Community-Based Experience after 60 Years of Pap Smear Usage,” Asian Pacific Journal Cancer Prevention, Vol. 13, No. 12, 2012, pp. 6497-6500.

http://dx.doi.org/10.7314/APJCP.2012.13.12.6497

[3] M. Maalej, K. Mrad, L. Kochbati, et al., “Cervical Cancer in Tunisia: An Epidemiological, Clinical and Pathological Study,” European Journal of Obstetrics \& Gynecology and Reproductive Biology, Vol. 113, No. 2, 2004, pp. 226-228. http://dx.doi.org/10.1016/j.ejogrb.2003.07.005

[4] M. Ben Laâbidi, "Evaluation de la Prévention des Cancers du col Utérin par le Frottis Cervico-Vaginal,” Maghreb Médical, Vol. 308, 1996, pp. 36-40.

[5] H. H. Nguyen. T. R. Broker and L. T. Chow, "Immune Responses to Human Papillomavirus in Genital Tract of Women with Cervical Cancer," Gynecologic Oncology, Vol. 96, No. 2, 2005, pp. 452-461.

http://dx.doi.org/10.1016/j.ygyno.2004.10.019 
[6] S. Arrossi, R. Sankaranarayanan and D. M. Parkin, "Incidence and Mortality of Cervical Cancer in Latin America," Salud Publica de Mexico, Vol. 45, No. 3, 2003, pp. 306-314.

[7] H. Zur Hausen, "Molecular Pathogenesis of Cancer of the Cervix and Its Causation by Specific Human Papillomavirus Types," Current Topics Microbiology and Immunology, Vol. 186, 1994, pp. 131-156.

[8] E. L. Franco, "Cancer Causes Revisited: Human Papillomavirus and Cervical Neoplasia," Journal of the National Cancer Institute, Vol. 87, No. 11, 1995, 779-780. http://dx.doi.org/10.1093/jnci/87.11.779

[9] N. Munoz, F. X. Bosch, S. de Sanjosé, et al., "International Agency for Research on Cancer Multicenter Cervical Cancer Study Group: Epidemiologic Classification of Human Papillomavirus Types Associated with Cervical Cancer,” The New England Journal of Medicine, Vol. 348, 2003, pp. 518-527. http://dx.doi.org/10.1056/NEJMoa021641

[10] J. S. Smith, L. Lindsay, B. Hoots, et al., "Human Papillomavirus Type Distribution in Invasive Cervical Cancer and High-Grade Cervical Lesions: A Meta-Analysis Update," International Journal of Cancer, Vol. 121, No. 3, 2007, pp. 621-632. http://dx.doi.org/10.1002/ijc.22527

[11] P. Sehr, K. Zumbach and M. Pawlita, “A Generic Capture ELISA for Recombinant Proteins Fused to Glutathione S-Transferase: Validation for HPV Serology," Journal of Immunological Methods, Vol. 253, No. 1-2, 2001, pp. 153-162.

http://dx.doi.org/10.1016/S0022-1759(01)00376-3

[12] W. Meschede, K. Zumbach, J. Braspenning, et al., “Antibodies against Early Proteins of Human Papillomavirus as Diagnostic Markers for Invasive Cervical Cancer,” Journal of Clinical Microbiology, Vol. 36, No. 2, 1998, pp. 475-480.

[13] L. Raymond, F. Menegoz, G. Fioretta, et al., "Recent Trends in Incidence of Cervical Cancer in Several Regions of Southern-Western Europe," Revue d'Epidémiolologie et de Santé Publique, Vol. 43, 1995, pp. 122-126.

[14] K. D. Hatch, A. Schnader and M. W. Abdel Nour, “An Evaluation of Human Papillomavirus Testing for Intermediate and High Risk Types as Triage before Colposcopy,” American Journal of Obstetrics and Gynecology, Vol. 172, No. 4, 1995, pp. 1150-1157.

http://dx.doi.org/10.1016/0002-9378(95)91473-0

[15] J. C. Boulanger, "Proposition d’Une Stratégie Thérapeutique,” Gynécologie, Vol. 38, 1987, pp. 397-403.

[16] T. B. Lawly, R. B. Lee and R. R. Kapela, "The Significance of Moderate and Severe Inflammation on Class I Papnicolaou Smear,” Obstetrics \& Gynecology, Vol. 76, No. 6, 1990, pp. 997-999.

[17] S. Cecchini, A. Iossa, S. Ciatto, et al., "Routine Colposcopic Survey of Patients with Squamous Atypia, a Method for Identifying Cases with False Negative Smears," Acta Cytological, Vol. 34, No. 6, 1990, pp. 778-780.

[18] S. Pairmuti, "False Negative Papanicolaou Smears from Women with Cancerous and Precancerous Lesions of the Uterine Cervix,” Acta Cytologica, Vol. 35, No. 1, 1991, pp. 40-70.
[19] G. Body, Ph. Descamps, J. Lansac, et al., "Néoplasies Intra Epithéliales du col. Encyclopédie Médico-Chirurgicale en Gynécologie 597-A-10, Cancérologie 60-200A-10. Paris,” Elsevier, 1993, p. 29.

[20] H. J. Soost, H. J. Lange and W. Lehmacher, “The Validation of Cervical Cytology Sensitivity and Predictive Valves,” Acta Ctytologica, Vol. 35, No. 1, 1991, pp. 8-14.

[21] D. C. Slawson, J. H. Bennett and J. H. Herman, "FollowUp to Papanicolaou Smear for Cervical Atypia Are We Missing Significant Disease? A Harnet Study,” Journal of Family Prost, Vol. 36, No. 3, 1993, pp. 289-293.

[22] S. R. Lindhein and G. Smith-Ngiyen, “Aggressive Evaluation for Atypical Squamous Cells in Papanicolaou Smears,” Journal of Reproductive Medicine, Vol. 35, No. 10, 1990, pp. 971-973.

[23] D. Dargent, "Valeur de la Cytologie Différentielle dans le Diagnostic des Formes Précliniques du Cancer CervicoUtérin,” Nov Pree Méd, Vol. 12, 1983, pp. 631-634.

[24] A. C. Pearlstone, P. W. Grigsby and D. G. Mutch, "High Rises of Atypical Cervical Cytology: Occurrence and Clinical Significance,” Obstetrics \& Gynecology, Vol. 80, 1992, pp. 191-195.

[25] G. L. Davis, E. Hernaudez, J. L. Davis, et al., "Atypical Squamous Cells in Papanicolaou Smears," Obstetrics \& Gynecology, Vol. 69, 1987, pp. 43-46.

[26] R. C. Reiter, "Management of Initial Atypical Cervical Cytology: A Randomized, Prospective Study," Obstetrics \& Gynecology, Vol. 68, No. 2, 1986, pp. 237-240.

[27] N. C. Seckin, et al., "Routine Evaluation of Patients Persistent Inflammatory Cellular Changes on Pap Smear," International Journal of Gynecology and Obstetrics, Vol. 59, No. 1, 1997, pp. 25-29. http://dx.doi.org/10.1016/S0020-7292(97)00113-6

[28] G. Sadoul and L. Beuret, “Analyse d'Une Serie Continue de 421 Condylomes gEnitaux,” Journal de Gynécologie Obstétrique et Biologie de la Reproduction, Vol. 14, No. 6, 1985, pp. 1049-1958.

[29] M. J. Campion, A. Singer and H. S. Mitchell, "Complacency in Diagnosis of Cervical Cancer,” British Medical Journal, Vol. 294, 1987, pp. 1337-1339. http://dx.doi.org/10.1136/bmj.294.6583.1337

[30] S. Kohan, J. Noumoff, E. M. Beckman, et al., "Colposcopic Screening of Women with Atypical Papanicolaou Smears,” Journal of Reproductive Medicine, Vol. 30, No. 5, 1985, pp. 383-387.

[31] I. Nyirjesy, "Atypical or Suspicious Cervical Smears," JAMA, Vol. 222, No. 6, 1972, pp. 691-693. http://dx.doi.org/10.1001/jama.1972.03210060045011

[32] H. F. Sandmire, S. D. Austin and R. C. Bechtel, "Experience with 40000 Papanicolaou Smears," Obstetrics \& Gynecology, Vol. 48, No. 1, 1976, p. 56.

[33] N. Spitzer, "Comparative Utility of Repes Papanicolaou Smears, Cervicography and Colposcopy in the Evaluation of Atypical Papanicolaou Smears,” Obstetrics \& Gynecology, Vol. 69, 1987, p. 731.

[34] S. Kohan, J. Noumoff, E. M. Beckman, et al., "Colposcopic Screening of Women with Atypical Papanicolaou Smears,” Journal of Reproductive Medicine, Vol. 30, No. 
5, 1985, pp. 383-387.

[35] G. N. Papanicolaou, "Atlas of Exfoliative Cytology," Harvard University Press, Cambridge, 1954, p. 450.

[36] M. Schiffman, P. E. Castle, J. Jeronimo, et al., "Human Papillomavirus and Cervical Cancer,” Lancet, Vol. 370, No. 9590, 2007, pp. 890-907. http://dx.doi.org/10.1016/S0140-6736(07)61416-0

[37] J. J. Carter, L. A. Koutsky, G. C. Wipf, et al., “The Natural History of Human Papillomavirus Type 16 Capsid Antibodies among a Cohort of University Women," Journal of Infectious Disease, Vol. 174, No. 5, 1996, pp. 927-936. http://dx.doi.org/10.1093/infdis/174.5.927

[38] P E. Castle, T. Shields, R. Kirnbauer, et al., "Sexual Behavior, Human Papillomavirus Type 16 (HPV 16) Infection, and HPV 16 Seropositivity," Sexually Transmitted Diseases, Vol. 29, No. 3, 2002, pp. 182-187. http://dx.doi.org/10.1097/00007435-200203000-00009

[39] D. Opalka, C. E. Lachman, S. A. MacMullen, et al., "Simultaneous Quantitation of Antibodies to Neutralizing Epitopes on Virus-Like Particles for Human Papilloma- virus Types 6, 11, 16, and 18 by a Multiplexed Luminex Assay," Clinical and Diagnostic Laboratory Immunology, Vol. 10, No. 1, 2003, pp. 108-115.

[40] J. L. Belinson, Y. L. Qiao, R. G. Pretorius, et al., "Shanxi Province Cervical Cancer Screening Study: A CrossSectional Comparative Trial of Multiple Techniques to Detect Cervical Neoplasia,” Gynecologic Oncology, Vol. 83, No. 2, 2001, pp. 439-444. http://dx.doi.org/10.1006/gyno.2001.6370

[41] J. L. Belinson, S. Hu, M. Niyazi, et al., "Prevalence of Type-Specific Human Papillomavirus in Endocervical, Upper and Lower Vaginal, Perineal, and Vaginal SelfCollected Specimens; Implications for Vaginal Self-Collection,” International Journal of Cancer, Vol. 127, No. 5, 2010, pp. 1151-1157. http://dx.doi.org/10.1002/ijc.25144

[42] M. Follen, S. Crain, C. MacAulay, et al., "Optical Technologies for Cervical Neoplasia; Update of an NCI Program Project Grant," Clinical Advances in Hematology and Oncology, Vol. 3, No. 1, 2005, pp. 41-53. 